\title{
INVARIANT MEASURES FOR AFFINE FOLIATIONS
}

\author{
WILLIAM M. GOLDMAN, MORRIS W. HIRSCH AND GILBERT LEVITT
}

\begin{abstract}
A (transversely) affine foliation is a foliation with an atlas whose coordinate changes are locally affine. Such foliations arise naturally in the study of affine structures on manifolds. In this paper we prove that an affine foliation with nilpotent affine holonomy group always admits a nontrivial transverse measure. Two proofs are given: one for noncompact manifolds, and another, valid for compact manifolds with $(G, X)$ )-foliations (not necessarily affine) having nilpotent holonomy. These results are applied to prove that a certain cohomology class on a compact affine manifold with nilpotent holonomy is nonzero. Examples are discussed.
\end{abstract}

Introduction. A smooth foliation of a manifold $M$ is a partition of $M$ into submanifolds (leaves) together with a transverse differentiable structure. In the presence of a more rigid transverse structure (e.g. an affine structure, or more generally a geometric structure), one may infer more detailed information on the possible properties of the foliation (for example, the existence of a transverse measure).

To illustrate this idea, we shall first study (transversely) affine foliations and prove

THEOREM A. Let $\mathcal{\mp}$ be an affine foliation of a manifold $M$. If the (affine) holonomy group of $\mp$ is virtually nilpotent, then there exists a transverse measure for 于.

A group if virtually nilpotent if it contains a nilpotent subgroup of finite index. Note that if the fundamental group $\pi_{1}(M)$ is (virtually) nilpotent then the holonomy group of $\mathcal{F}$ is as well.

This result is based on the following theorem, for which we give a constructive proof.

THEOREM B. Let $H$ be a virtually nilpotent group of affine transformations of $\mathbf{R}^{n}$. For any $H$-invariant open subset $U \subset \mathbf{R}^{n}$, there exists a nontrivial $H$-invariant Radon measure on $U$.

Recall that a Radon measure is a nontrivial Borel measure which is finite on compact sets.

For closed manifolds, Theorem $\mathrm{A}$ is a special case of a general result on $(G, X)$ foliations (i.e. foliations with a transverse geometric structure-see below).

THEOREM C. Let $₹$ be a $(G, X)$-foliation of a closed manifold $M$. If the holonomy group $\Gamma$ of $\mathcal{F}$ is virtually nilpotent then $\mathcal{F}$ admits a transverse measure.

Received by the editors August 18, 1981 and, in revised form, February 26, 1982.

1980 Mathematics Subject Classification. Primary 57R30; Secondary 58F11.

(c) 1982 American Mathematical Society 0002-9939/82/0000-0688/\$3.00 
This result follows from the work of Plante $[\mathbf{1 0}]$ and the fact that, for a $(G, X)$ foliation, the growth type of any leaf is dominated by the growth type of $\Gamma$ (a fortiori by the growth type of $\left.\pi_{1}(M)\right)$. In $\S 3$ we deduce it from the following theorem, which is a fairly straightforward consequence of results of Plante but has apparently never been explicitly stated.

THEOREM D. Let $H$ be a finitely generated group acting on a locally compact space $Y$ such that some compact subset of $Y$ meets every orbit. If the orbit Hy of a point $y \in Y$ has nonexponential growth (in particular, if $H$ is virtually nilpotent), there exists an $H$-invariant Radon measure on $Y$ whose support is contained in $\overline{H y}$.

These results will be applied to the study of closed affine manifolds with nilpotent holonomy. If $M$ is such a manifold, then there is a natural pair of transverse foliations of $M$ by parallel affine submanifolds. For one of these foliations there is a transverse parallel differential form $\omega$ defining a smooth transverse measure. Applying Theorem A, we prove in $\S 4$,

THEOREM E. The cohomology class of $\omega$ in $H^{*}(M ; R)$ is nonzero.

In contrast we give examples of nonzero parallel differential forms on compact affine manifolds, and of exterior forms transverse to affine foliations of compact manifolds, which are exact.

We thank the referee for the following generalization of Theorems A and B. The referee's proof is given in $\S 3$.

THEOREM R. Let $G$ be a real algebraic group acting algebraically on a variety $X$.

(a) If a $(G, X)$-foliation $₹$ of a manifold $M$ has virtually nilpotent holonomy, then $\mp$ has a transverse measure.

(b) If a virtually nilpotent subgroup $\Gamma \subset G$ leaves invariant an open set (in the Hausdorff topology) $U \subset X$ then $\Gamma$ preserves a Radon measure on $U$.

REMARK. Not every nilpotent action preserves a Radon measure. In fact, M. Herman has constructed a diffeomorphism of a noncompact surface having no invariant Radon measure.

1. Definitions. In what follows, $M$ denotes a connected closed $n$-manifold and $\exists$ is a codimension- $q$ foliation of $M$. Let $X$ be a connected $q$-manifold upon which a group $G$ acts. We shall always assume that no element of $G$, except the identity, fixes a nonempty open set in $X$. For example, $X$ could be a finite dimensional real vector space $E$ and $G$ the group $\operatorname{Aff}(E)$ of affine transformations of $E$.

A $(G, X)$-structure on a foliation $\mathcal{F}$ is a maximal atlas $\left\{\left(U_{\alpha}, \psi_{\alpha}\right)\right\}$ of submersions $\Psi_{\alpha}: U_{\alpha} \rightarrow X$ defining $₹$ such that for every component $C$ of $U_{\alpha} \cap U_{\beta}$ there is a transformation $f=f_{\alpha, \beta, C} \in G$ making the following diagram commute.

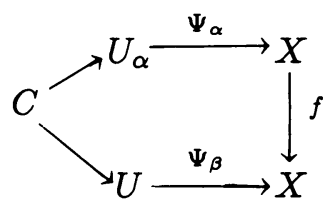


REMARKS. (1) When $\mathcal{F}$ is the foliation of $M$ by points, a $(G, X)$-structure on $\mp$ is precisely a $(G, X)$-structure on $M$. For more details on $(G, X)$-structures see Thurston [15], Goldman [4] or Kulkarni [9].

(2) A $(G, X)$-structure on $₹$ induces a $(G, X)$-structure on any $q$-dimensional submanifold transverse to $\mathcal{F}$.

(3) When $(G, X)=(\operatorname{Aff}(E), E)$ we simply speak of affine structures.

It is well known (see, e.g., Goldman [4] or Thurston [15]) that every $(G, X)$ foliation can be constructed as follows. First let $\tilde{M} \rightarrow M$ be a universal covering with group of covering transformations $\pi=\pi_{1}(M)$. Suppose $D: \tilde{M} \rightarrow X$ is a submersion which is equivariant with respect to a homomorphism $\phi: \pi \rightarrow G$. The submersion $D$ defines a codimension- $q$ foliation $\tilde{\mathcal{F}}$ on $\tilde{M}$ which has a canonical $(G, X)$-structure. This $(G, X)$-foliation is invariant under $\pi$ and therefore descends to a $(G, X)$-foliation $\mathcal{F}$ on $M$. The map $D$ is called a developing map for $₹$ and $\phi$ is the $(G, X)$-holonomy homomorphism. The image of $\phi$ is the holonomy group of $\mathcal{F}$ and will henceforth be denoted $\Gamma$.

It is a general principle that $\Gamma$-invariant "objects" defined on the developing image $D(\tilde{M})$ pull back to $\pi$-invariant "objects" on $\tilde{M}$, which in turn define "objects" on $M$. We shall apply this principle to foliations, tensor fields and measures. In particular any $\Gamma$-invariant Radon measure on $D(\tilde{M})$ defines a transverse measure on $\mathcal{F}$ in the sense of Plante [10] and Ruelle and Sullivan [12].

2. Nilpotent groups of affine transformations. The purpose of this section is to present a direct proof of the following result.

Proposition 2.1. Let $E$ be a finite dimensional real vector space and $H$ a virtually nilpotent group of affine transformations of $E$. Suppose $U \subset E$ is an $H$ invariant open subset. Then there exists a nontrivial $H$-invariant Radon measure $\mu$ on $U$ and a finite union $S(U) \subset U$ of sectors (see below) such that $\mu(V)>0$ whenever $V$ is an open subset of $U$ meeting $S(U)$ and $\mu(A)=0$ whenever $A$ is disjoint from $S(U)$.

A sector of $U$ is a subset of the form

$$
U \cap\left\{x \in E: f_{1}(x)=\cdots=f_{q}(x)=0, f_{q+1}(x)>0, \ldots, f_{s}(x)>0\right\}
$$

where $f_{1}, \ldots, f_{s}$ are affine maps $E \rightarrow \mathbf{R}$ whose linear parts are linearly independent.

Proposition 2.1 contains Theorem B, and also implies Theorem A (take $U=$ $D(\tilde{M})$ and $H=\Gamma)$.

Our basic tool in the proof of Proposition 2.1 will be the following pair of structure theorems for affine representations of nilpotent groups (see Fried, Goldman and Hirsch [2]). For any subgroup $H \subset \operatorname{Aff}(E)$ we denote by $L(H) \subset \mathrm{GL}(E)$ its linear part, i.e., the image of $H$ under the homomorphism $\operatorname{Aff}(E) \rightarrow \operatorname{GL}(E)$ which to the affine transformation $x \mapsto A x+b$ assigns the linear transformation $A$.

Proposition 2.2. Let $H$ be a nilpotent subgroup of $\operatorname{Aff}(E)$.

(a) (Fitting splitting) Let $E_{U} \subset E$ be the maximal $L(H)$-invariant linear subspace on which $L(H)$ acts unipotently. Then $H$ leaves invariant a unique coset of $E_{U}$ and $L(H)$ leaves invariant a unique complementary linear subspace $F$. Then $E$ has the $L(H)$-invariant splitting $E_{U} \oplus F$. Moreover $H$ permutes the cosets of $E_{U}$, and of $F$; the induced affine action of $H$ on $E / E_{U}$ has a unique stationary point. This induced 
action is conjugate via a translation to a linear action of $H$ on $E / E_{U}$, and thus to the linear action of $L(H)$ on $F$.

(b) (Primary decomposition) Suppose that $H \subset \mathrm{GL}(E)$. Then there exists a subgroup $H_{1} \subset H$ of finite index in $H$, an $H_{1}$-invariant linear decomposition

$$
E=\left(\bigoplus_{i=1}^{r} E_{i}\right) \oplus\left(\bigoplus_{i=r+1}^{r+c}\right) E_{i}
$$

(where each $E_{i}$ is endowed with a complex structure for $r+1 \leq i \leq r+c$ ) and homomorphisms $\lambda_{i}: H_{1} \rightarrow \mathbf{R}^{*}(i=1, \ldots, r), \lambda_{i}: H_{1} \rightarrow \mathbf{C}^{*}(i=r+1, \ldots, r+c)$, such that $\left(h-\lambda_{i}(h) I\right)$ acts unipotently on $E_{i}$ for each $h \in H_{1}$. (Here $I$ denotes the identity transformation on $E_{i}$.)

Proof of Proposition 2.1. First we observe that if Proposition 2.1 is true for a subgroup $H^{\prime} \subset H$ of finite idex then it is also true for $H$ : we simply average an $H^{\prime}$-invariant measure over the right cosets of $H^{\prime}$ in $H$. This allows us to pass to subgroups of finite index whenever convenient. In particular we can assume that $H$ is nilpotent.

We first consider the special case where $H$ is linear. We thus restrict attention to nilpotent subgroups $H$ of $\mathrm{GL}(E)$ which admit a primary decomposition as in 2.2(b). Since $h-\lambda_{i}(h) I$ acts unipotently on $E_{i}$ for each $h \in H$, there exists a nonzero linear functional $u_{i}: E_{i} \rightarrow \mathbf{R}$ (respectively, $u_{i}: E_{i} \rightarrow \mathbf{C}$ ) such that $u_{i}(h x)=\lambda_{i}(h) u_{i}(x)$ for all $h \in H, x \in E_{i}$.

We proceed by induction on $n=\operatorname{dim} E$, noting that the case $\operatorname{dim} E=0$ is trivial. Assume the result has been proved for spaces of dimension less than $n$. Let $F_{i} \subset E_{i}$ be the kernel of $u_{i}$; let $p_{i}: E \rightarrow E_{i}$ be the projection of the primary decomposition. Each $p_{i}^{-1}\left(F_{i}\right)$ is an $H$-invariant subspace of $E$ having dimension strictly less than $n$.

First suppose that some $p_{i}^{-1}\left(F_{i}\right)$ meets $U$. By the induction hypothesis there exists a nonzero $H$-invariant Radon measure $\mu_{i}$ on $U \cap p_{i}^{-1} F_{i}$ satisfying 2.1. Define $\mu$ by $\mu(A)=\mu_{i}\left(A \cap F_{i}\right)$ for Borel sets $A \subset U$. Clearly $\mu$ is a Radon measure on $U$ satisfying 2.1 .

We can therefore assume that $U$ is disjoint from each set $p_{i}^{-1}\left(F_{i}\right)$; thus $U \subset$ $\prod_{i=1}^{r+c}\left(E_{i}-F_{i}\right)$ (Cartesian product). Let $\left|d x_{i}\right|$ denote Lebesgue measure on $E_{i}$. Set $\operatorname{dim}_{\mathbf{R}} E_{i}=k_{i}$. Then

$$
\mu_{i}=\left|u_{i}(x)\right|^{-k_{i}}\left|d x_{i}\right|
$$

defines an $H$-invariant Radon measure on $E_{i}-F_{i}$. The product measure $\prod_{i} \mu_{i}$ is an $H$-invariant Radon measure on $\prod_{i}\left(E_{i}-F_{i}\right)$ whose restriction to $U$ satisfies 2.1.

Now consider the general case $H \subset \operatorname{Aff}(E)$. Let $E=E_{U} \oplus F$ be the Fitting decomposition for the action of $H$ on $E$. Let $p: E \rightarrow E / E_{U}$ be the canonical projection.

By 2.2(a) we may assume that $H$ leaves $E_{U}$ invariant and thus induces a linear action on $E / E_{U}$. Therefore the special case yields an $H$-invariant measure $\mu_{F}$ on $p(U)$ supported in a finite union of sectors. As $L(H)$ acts unipotently on $E_{U}$, the Lebesgue measure $\mu_{U}$ on $E_{U}$ is $H$-invariant and the restriction to $U$ of the "product" measure $\mu_{F} \times \mu_{U}$ clearly satisfies the conclusions of 2.1 (given that $\mu_{F}$ does relative to $p(U))$. Q.E.D. 
3. Existence of invariant measures. Say that a group action on a locally compact space is syndetic (Gottschalk and Hedlund [7]) if there exists a compact set which meets every orbit. Theorem D can then be rephrased as follows.

THEOREM D. Let $H$ be a finitely generated group acting syndetically on a locally compact space $Y$. If the orbit $H y$ of a point $y \in Y$ has nonexponential growth, there exists a nontrivial $H$-invariant Radon measure on $Y$ whose support is contained in $\overline{H y}$.

This theorem is a corollary of Theorem 3.1 and Lemma 3.2 of Plante [10]. Note that by using Plante's techniques one can give a proof of Theorem $\mathrm{D}$ that does not use the notion of pseudogroup and yields directly a countably additive measure.

REMARKS. If $H$ is virtually nilpotent, then every orbit has nonexponential growth.

There are counterexamples to Theorem D for nonsyndetic actions of the group of integers on $R^{2}-\{0\}$.

Here is how to deduce Theorem $\mathrm{C}$ from Theorem $\mathrm{D}$.

Proof of TheOREM C. Let $₹$ be a $(G, X)$-foliation of a closed manifold $M$, and let $\Gamma$ be the holonomy group. Since $M$ is compact the action of $\Gamma$ on $D(\tilde{M})$ is syndetic. As $\Gamma$ is assumed to be virtually nilpotent, every orbit of $\Gamma$ in $D(\tilde{M})$ has nonexponential growth, and Theorem $\mathrm{D}$ yields a $\Gamma$-invariant Radon measure on $D(\tilde{M})$. Therefore $\mathcal{F}$ admits a nontrivial transverse measure. Q.E.D.

REMARK. If $D: \tilde{M} \rightarrow X$ is not a fibration onto its image, there may exist transverse measures of $₹$ which do not come from $\Gamma$-invariant measures on $D(\tilde{M})$. For a simple example we may start from a transversely affine Anosov foliation on a hyperbolic torus bundle over $S^{1}$ and "tubularize" it, creating a compact leaf. The resulting foliation is affine and possesses an atomic transverse measure, but $\Gamma$ preserves no Radon measures on $D(\tilde{M})=R$.

One can construct examples, using (real and complex) projective structures on surfaces of genus $>1$ whose developing map is onto projective space but whose holonomy group preserves no measure on $D(\tilde{M})$. See, e.g., Sullivan and Thurston [14] or Hejhal [8].

Theorem $\mathrm{C}$ can also be deduced from Theorem 4.1 of Plante [10], using the following fact proved by R. A. Blumenthal [16].

LEMMA. Let $\mathcal{F}$ be a $(G, X)$-foliation of a closed manifold $M$, with developing map $D: \tilde{M} \rightarrow X$ and holonomy group $\Gamma \subset G$. If $\tilde{x} \in \tilde{M}$ projects down to $x \in M$, the growth type (in the sense of [10]) of the leaf of $\mp$ through $x$ is dominated by the growth type of the orbit of $D(\tilde{x})$ under $\Gamma$.

COROLlARY. For a $(G, X)$-foliation of a closed manifold $M$, the growth type of any leaf is dominated by the growth type of $\pi_{1}(M)$.

PROOF OF LEMMA. Choose a regular covering of $M$ (in the sense of $[10, p$. 337]) by open sets $U_{i}(1 \leq i \leq q)$ such that $U_{i} \cup U_{j}$ is contained in a ball whenever $U_{i}$ and $U_{j}$ intersect. As in [10], for each $i$, we can define the space $X_{i}$ of plaques of $U_{i}$ (identified with $\psi_{i}^{-1}\left(D^{k} \times\{0\}\right)$ ), and the transition functions $\gamma_{i j}$. Choose local developing maps $D_{i}: U_{i} \rightarrow X$. If $U_{i} \cap U_{j}$ is nonempty, the transition function $\gamma_{i j}$ is defined; because $U_{i} \cup U_{j}$ is contained in a ball, there is a unique element $h_{i j}$ in $\Gamma$ such that $D_{j}\left(\gamma_{j i} x\right)=h_{j i} D_{i}(x)$ for $x \in X_{i}$ in the domain of $\gamma_{j i}$. By induction on $k$ 
we get $D_{i_{k}}\left(\gamma_{i_{k} i_{k-1}} \cdots \gamma_{i_{2} i_{1}} x\right)=h_{i_{k} i_{k-1}} \cdots h_{i_{2} i_{1}} \cdot D_{i_{1}}(x)$ whenever the left-hand side is defined.

We can assume that $x \in X_{1}$ and $D_{1}(x)=D(\tilde{x})$. To a chain of plaques $\left(\rho_{1}, \ldots, \rho_{k}\right)$ $\subset X_{1} \times X_{i_{2}} \times \cdots \times X_{i_{k}}$ starting at $x$ we can associate the point $h_{i_{k} i_{k-1}} \cdots h_{i_{2} 1} \cdot D_{1}(x)$ in $D(\tilde{M})$; this point depends only on the end plaque in the chain, and because $D_{k}$ is injective two chains ending at different plaques in the same $X_{i_{k}}$ give rise to different points in $D(\tilde{M})$. This shows that the growth function of the leaf of $₹$ through $x$ is dominated by $q$ times the growth function of the orbit of $D_{1}(x)=D(\tilde{x})$ under the subgroup of $\Gamma$ generated by the elements $h_{i j}$. This proves the lemma. Q.E.D.

Proof of Theorem R. Let $A$ denote the Zariski closure of $\Gamma$ in $G$. The closure of each $A$-orbit $A x$ in $S$ is the union of $A x$ together with $A$-orbits of smaller dimension. Let $I_{x} \subset A$ denote the isotropy group of $x \in X$ in $A$. Then the orbit $A x$ of $x$ under $A$ is equivariantly homeomorphic to the left coset space $A / I_{x}$.

Fix $x \in U$ so that $A x$ has minimal dimension of all $A$-orbits meeting $U$. Since $A$ is a nilpotent algebraic group $A / I_{x}$ carries an $A$-invariant Radon measure. Consequently $A x$ carries an $A$-invariant Radon measure $\mu_{0}$. Define an $A$-invariant measure $\mu$ on $U$ by $\mu(s)=\mu_{0}(s \cap A x)$. Since $A x$ has minimal dimension it is closed in the relative Hausdorff topology on $U$. This implies that $\mu$ is a Radon measure, proving (b).

The derivation of (a) from (b) is the same as that of Theorem $\mathrm{C}$ from Theorem D. Q.E.D.

It is interesting to note that the proofs of Proposition 2.1 and Theorem $\mathrm{R}$, when applied to a nilpotent group of affine transformations, can give different invariant measures. For example if

$$
\Gamma=\left\{\exp \left[\begin{array}{cc}
a t & 0 \\
0 & b t
\end{array}\right]: t \in \mathbf{R}\right\}
$$

and

$$
U=\left\{(x, y) \in \mathbf{R}^{2}: x>0, y>0\right\}
$$

then Proposition 2.1 gives the invariant Radon measure $d x / x \cdot d y / y$ whose support is all of $U$. But if $a$ and $b$ are both integers, then the proof of Theorem $\mathrm{R}$ gives a measure whose support is 1-dimensional.

4. Applications to affine manifolds. Let $M$ be a closed oriented affine manifolds with nilpotent holonomy group $\Gamma$. Denote by $E_{U}$ the Fitting subspace for $\Gamma$ as in 2.2(a) and let $p: E \rightarrow E_{U}$ be the projection with kernel $F$, invariant under $L(\Gamma)$. Since $\Gamma$ acts unipotently on $E_{U}$ the Euclidean volume form $\omega_{U}$ on $E_{U}$ is preserved. It follows that $p^{*} \omega_{U}$ defines a $\Gamma$-invariant parallel $k$-form on $E$ (where $k=\operatorname{dim} E_{U}$ ), and it therefore defines a parallel $k$-form $\omega_{M}$ on $M$.

THEOREM E. The cohomology class $\left[\omega_{M}\right] \in H^{k}(M ; \mathbf{R})$ is nonzero.

After proving Theorem $\mathrm{E}$ we shall give several examples of cohomologically trivial parallel forms which are transverse to an affine foliation of complementary dimension.

PROOF OF THEOREM E. Let $\mathcal{F}_{F}(E)$ denote the foliation on $E$ by cosets of $F$, and $\mathcal{F}_{U}(E)$ the foliation by cosets of $E_{U}$. These foliations are $\Gamma$-invariant and thus induce a transverse pair of foliations $\mathcal{F}_{F}, \mathcal{F}_{U}$ of $M$. The holonomy of the affine foliation $F_{U}$ can be identified with the nilpotent group $L(\Gamma)$ acting on $E / E_{U}$. By 
Theorem $\mathrm{A}$ there is an invariant transverse measure $\mu$ for $\mathcal{F}_{U}$. Also the parallel form $\omega_{M}$ defines a transverse measure $\nu$ for $\mathcal{F}_{F}$.

Choose a transverse orientation for $\mathcal{F}_{U}$. The measure $\mu$, together with the orientation, determines a cohomology class $[\mu] \in H^{n-k}(M ; \mathbf{R})$. The pair of foliations $\mathcal{F}_{U}, \mathcal{F}_{F}$ intersect transversely and the foliation $\mathcal{F}_{U} \cap \mathcal{F}_{F}$, whose leaves are connected components of intersection of leaves of $\mathcal{F}_{U}$ with leaves of $\mathcal{F}_{F}$, is the foliation of $M$ by points. As in Ruelle and Sullivan [12] there is a "product measure" $\mu \times \nu$ on $\mathcal{F}_{U} \cap \mathcal{F}_{F}$ defined as follows: If $T_{U}$ is an $\mathcal{F}_{U}$-transversal and $T_{F}$ is an $\mathcal{F}_{F}$-transversal, then $T_{U} \times T_{F}$ is a transversal to $\mathcal{F}_{U} \cap \mathcal{F}_{F}$ and $(\mu \times \nu)\left(T_{U} \times T_{F}\right)$ is just the product measure $\mu\left(T_{U}\right) \times \nu\left(T_{F}\right)$. There is a unique extension of $\mu \times \nu$ from transversals of the form $T_{U} \times T_{F}$ to arbitrary transversals of $\mathcal{F}_{U} \cap \mathcal{F}_{F}$. Moreover, if $\mathcal{F}_{U} \cap \mathcal{F}_{F}$ is given the transverse orientation determined by those of $\mathcal{F}_{U}$ and $\mathcal{F}_{F}$, the cohomology class $[\mu \times \nu]$ is just the cup-product $[\mu]\left[\omega_{M}\right]$.

Since $\mathcal{F}_{U} \cap \mathcal{F}_{F}$ is the foliation of $M$ by points, the transverse measure $\mu \times \nu_{M}$ is a measure of $M$. It is clearly a Radon measure. Moreover the evaluation of $[\mu \times \nu]$ on the fundamental class $[M] \in H_{n}(M ; \mathbf{R})$ is (up to sign) the total mass $\int_{M}(\mu \times \nu)$, which is positive. Hence $[\mu]\left[\omega_{M}\right]=[\mu \times \nu]$ is nonzero in $H^{n}(M ; \mathbf{R})$ and therefore $[\omega]$ is nonzero in $H^{k}(M ; \mathbf{R})$. Q.E.D.

EXAMPLES. (1) let $\Gamma$ be the nilpotent group consisting of all affine transformations

$$
\left[\begin{array}{ccc}
1 & -u & t \\
0 & 1 & 0 \\
0 & 0 & 1
\end{array}\right]\left[\begin{array}{l}
s \\
t \\
u
\end{array}\right]
$$

where $s, t, u$ are integers. One can easily prove that $\Gamma$ acts properly discontinuously and freely on $E=\mathbf{R}^{3}$ and the quotient $M=E / \Gamma$ is a compact complete affine nilmanifold (see [3] for the definition). Moreover, the contact form $d x-z d y+y d z$ on $E$ is $\Gamma$-invariant and defines an affine 1 -form $\theta$ on $M$. Then $d \theta$ is a nonzero parallel exterior 2 -form on $M$, which is exact even though it is "transverse" to the affine foliation defined by the parallel vector field $\partial / \partial x$.

(2) In $[3, \S 6]$ an example is given of an affine structure on a hyperbolic $T^{2}$-bundle over $S^{1}$ which has both a radiant vector field $R$ (in local coordinates $R=x \partial / \partial x+$ $y \partial / \partial y+z \partial / \partial z)$ and a parallel 2-form $\omega$. Let $\iota_{R} \omega$ denote the interior product of $R$ and $\omega$. Then $\omega=d \iota_{R} \omega$ and thus the cohomology class $[\omega]$ is zero. Moreover it is easy to see that $\omega$ defines an area form transverse to a parallel line field. Compare also [5].

(3) Here is an example of an affine foliation which does not arise from an affine structure on a manifold, and which has a transverse parallel volume form $\omega$ which is zero in cohomology. Let $\operatorname{SL}(2, \mathbf{R})$ act on $\mathbf{R}^{2}-\{0\}$ in the standard way, and on itself by left-multiplication. The evaluation map $f: \operatorname{SL}(2, \mathbf{R}) \rightarrow \mathbf{R}^{2}-0$ at any $x_{0} \in \mathbf{R}^{2}-\{0\}$ is an $\operatorname{SL}(2, \mathbf{R})$-equivariant fibration. It defines a codimension-2 affine foliation on $\operatorname{SL}(2, \mathbf{R})$ which is invariant under left multiplication. Thus it defines an affine foliation $₹$ of $M=\Gamma \backslash \mathrm{SL}(2, \mathbf{R})$ for any discrete cocompact subgroup $\Gamma \subset$ $\operatorname{SL}(2, \mathbf{R})$. The Euclidean area form $d x \wedge d y$ on $\mathbf{R}^{2}-\{0\}$, which is invariant under $\operatorname{SL}(2, \mathbf{R})$, defines a transverse parallel area from $\eta$ to $\mathcal{F}$. The cohomology class $[\eta] \in$ $H^{2}(M ; \mathbf{R})$ is zero for the following reason. The radial vector field $x \partial / \partial x+y \partial / \partial y$ on $\mathbf{R}^{2}-\{0\}$ defines a nonsingular vector field $R$ on $M$ which is transverse to $\mathcal{F}$. 
Moreover it is easy to check in local coordinates that $d\left(\iota_{R} \eta\right)=\eta$ so that $[\eta]=0$ as desired.

\section{REFERENCES}

1. E. Fédida and P. Furness,Feuilletages transversalement affines de codimenson 1, C. R. Acad. Sci. Paris 282 (1976), 825-827.

2. D. Fried, W. Goldman and M. Hirsch, Affine manifolds with nilpoltent holonomy, Comm. Math. Helv. 56 (1981) (to appear).

3. P. Furness and E. Fédida, Transversely affine foliations, Glasgow Math. J. 17 (1976), 106-111.

4. W. Goldman, Discontinuous groups and the Euler class, $\S 1$, Ph.D. Thesis, University of California, Berkeley, 1980.

5. W. Goldman and M. Hirsch, Polynomial forms on affine manifolds, Pacific J. Math. (to appear).

6. _. Parallel characteristic class of affine manifolds, (in preparation).

7. W. Gottschalk and G. Hedlund, Topological dyamics, Amer. Math. Soc. Colloq. Publ., vol. 36, Amer. Math. Soc., Providence, R.I., 1965.

8. D. Hejhal, Monodromy groups and linearly polymorphic functions, Acta Math. 135 (1975), 1-55.

9. R. Kulkarni, On the principle of uniformization, J. Differential Geom. 13 (1978), 109-138.

10. J. Plante, Foliations with measure-preserving holonomy, Ann. of Math. 102 (1975), 327-361.

11. Solvable group actions on the line, University of North Carolina, 1980 (preprint).

12. D. Ruelle and D. Sullivan, Currents, flows, and diffeomorphisms, Topology 14 (1975), 319-327.

13. B. Seke, Sur les structures transversalement affines des feuilletages de codimension un, Ann. Inst. Fourier (Grenoble) 30 (1980), 1-30.

14. D. Sullivan and W. Thurston, Manifolds with canonical coordinates: some examples, Inst. Hautes Etude Sci. Publ. Math., 1979 (preprint).

15. W. Thurston, The geometry and topology of three-manifolds, Chapter 4, Princeton Univ. Press, Princeton, N.J., 1979 (preprint).

16. R. A. Blumenthal, Transversely homogeneous foliations, Ann. Inst. Fourier (Grenoble) 29 (1979), 143-158.

Department of Mathematics, Massachusetts institute of TeChNology, CAMBRIDGE, MASSACHUSETTS 02139

Department of Mathematics, University of California, Berkeley, CaliFORNIA 94720

Département de Mathématiques, Université Paris VII, Paris, France 